\title{
Clinical Features and Outcomes of Raoultella Terrigena Infections. A Single-center Experience from Karachi, Pakistan: A Retrospective Study
}

Ishfaque Ahmed

Aga Khan University Hospital

Nosheen Nasir

Aga Khan University Hospital

Bushra Jamil

Aga Khan University Hospital

Syed Faisal Mahmood ( $\square$ faisal.mahmood@aku.edu )

Aga Khan University Hospital

\section{Research Article}

Keywords: "Raoultella terrigena," "Klebsiella terrigena," "Raoultella species," "Klebsiella species"

Posted Date: January 4th, 2021

DOI: https://doi.org/10.21203/rs.3.rs-129254/v1

License: (c) (i) This work is licensed under a Creative Commons Attribution 4.0 International License. Read Full License 


\section{Abstract}

\section{Objectives:}

Raoultella terrigena (formerly Klebsiella terrigena) is an environmental gram-negative rod. It may cause infections in humans, especially in the elderly and immunosuppressed patients. Moreover, this organism tends to be multi-drug resistant, limiting treatment options. Evidence on clinical presentation and outcomes of this infection is limited. We conducted this study to see its various clinical features, available treatment options, and associated clinical impacts of the R.terrigena infections.

\section{Methodology:}

We conducted a cross-sectional study on all adult patients with clinical specimens positive for Raoultella terrigena at a 700-bedded tertiary care hospital in Karachi, Pakistan, between January 2013 to December 2018.

\section{Results:}

There were 58 patients with R.terrigena in different cultures specimens identified. Amongst them $n=12$ $(22.6 \%)$ were colonizers. The median age was 61.5 years ( $\mathrm{IQR}=28)$, and most were male $(60.9 \%)$. The most common infection site was the respiratory tract in $28.3 \%$, followed by urinary tract infections, $26 \%$, and central line-associated infections at $26.1 \%$. Amongst infected cases, $37 \%$ were in septic shock, and of which $76.5 \%$ required vasopressors. $45.7 \%$ had respiratory failure requiring non-invasive ventilation in $47.6 \%$, and mechanical ventilation in $52.4 \%$. The previous history of multidrug-resistant organisms was present in $67.4 \%$ of patients and the history of prior antibiotics use within the last six months was present in $78.3 \%$. $91.3 \%$ of isolates were resistant to piperacillin-tazobactam and meropenem, $65.2 \%$ were resistant to colistemethate. Eight patients lost to follow up. The majority of patients treated with combination therapy $(n=31,81.6 \%)$ and meropenem plus colistemethate was the most commonly used combination $(n=11,44 \%)$. The overall mortality rate was $44.7 \%$.

\section{Conclusion:}

R.terrigena is a highly drug-resistant organism with a high mortality rate and causes hospital-acquired respiratory tract infections in most patients.

\section{Background}

Raoultella Terrigena, initially known as "Klebsiella Terrigenna," was discovered in 1981 and is a rare gramnegative organism, mostly found in soil and water ${ }^{1}$. It was separated from Klebsiella species in 2001 based on molecular analysis ${ }^{2}$. These organisms are oxidase negative, capsulated, immotile, facultatively anaerobic, and aerobic bacilli ${ }^{2}$. However, many microbiology laboratories continue to identify this organism as a "Klebsiella species," making the true incidence and pathogenesis uncertain ${ }^{3}$. The first 
reported case as a human pathogen was in 2007 in a middle-aged post-liver transplant patient with endocarditis ${ }^{4}$. Another case report was published on sepsis secondary to $R$. terrigena in $2011^{5}$. A literature search reveals that most of the reported cases caused by genus Raoultella consist of $R$. orinithinolytica and R.planticola ${ }^{6,7}$, and are associated with diseases of the biliary tract and surgical interventions ${ }^{6,8}$ as well as necrotizing fasciitis ${ }^{9}$, cellulitis ${ }^{10}$, cystitis ${ }^{11}{ }^{\text {, cholangitis }}{ }^{12}$, cholecystitis ${ }^{13}$, and pancreatitis ${ }^{14}$. However, despite reviewing the literature, we could not find a sizeable descriptive study on clinical presentations and outcomes on Raoultella terrigena.

\section{Ethical review:}

The study started after receiving approval for exemption from the Aga Khan University Ethics review committee. (ERC Reference No:2019-1232-3287). Patient confidentiality was maintained by not taking personal identifiers such as names or medical record numbers. As this was a retrospective study, the committee waived the requirement of informed consent.

\section{Methodology}

We conducted a cross-sectional study on all adults above 18 years of age, admitted to Aga Khan University Hospital (AKUH) between (January 2013 to December 2018), who had Raoultella terrigena in cultures. We excluded patients with recurrent Raoultella infections and colonizers. Patients who fulfilled the eligibility criteria were consecutively included in the study. Data on patient-related variables such as age, sex, co-morbid, prior history of hospitalizations, invasive procedures, and use of antibiotics, etc., were collected from the Health care information and management system (HIMS) on a pre-tested structured proforma. Microbiological culture specimens related data extracted from Integrated Laboratory Management Services records.

\section{Laboratory Identification}

The identification process was performed in the microbiology laboratory at Aga Khan University Hospital Karachi. This involved culture inoculation on MacConkey, chocolate, and 5\% sheep blood agar at 37 degrees Celsius for up to 48 hours. The blood agar was made by phlebotomizing sheep in the animal house at AKUH. Upon growth of lactose fermenter mucoid colonies on MacConkey agar, further biochemical tests involve the utilization of citrate, hydrogen sulfide, urease, indole, and motility tests negative within acidic/acidic reaction on Tripe Sugar Test.

Further identification was carried out by API 20 E, which detects $91 \%$ to $95 \%$ of cases correctly. Antibiotic susceptibility testing was initially carried out by Mueller-Hinton agar with Kirby-Bauer disk diffusion test or Vitek-2 MSautomatedc system. Colistin minimum inhibitory concentrations were confirmed by colistin broth microdilution, which is currently the most reliable method. Results were interpreted as per Clinical and Laboratory Standards Institute antibiotic guidelines. 


\section{Statistical Methods}

Descriptive analysis was performed for all patient-related variables with frequencies and proportions reported for categorical variables like sex, comorbid, clinical features, etc. And median with interquartile range reported for continuous variables like age, hospital stay. Chi-square test or Fisher exact test were used as appropriate to determine the association between two categorical variables, e.g., chronic kidney disease and death. SPSS software (version 25.0) was used for data analysis. A P-value of less than 0.05 is supposed to be statistically significant.

\section{Results}

There were 58 patients with R.terrigena isolated from different culture specimens. Out of those, 12 patients were identified as colonizers, remaining included in the study (see Figure-1). The median age was 61.5 years (IQR=28), with more males than females $(60.9 \%$ vs. $39.1 \%$.) The most frequent co-morbid conditions were diabetes mellitus in $n=18$ (39.1\%), followed by chronic kidney disease in $n=16(34.7 \%)$ and malignancy in $n=11$ (23.9) (see table-1). Most patients $n=34(73.9 \%)$ had a previous history of hospitalization (within the past six months), and in the majority, $n=24(52.17 \%)$ had at least one hospital admission. $78 \%$ patients $(n=36)$ reported antibiotic use in last 6 months. The most commonly used antibiotics were carbapenems in $n=26(56.5 \%)$. Also beta-lactam, beta-lactamase inhibitors in $n=23(50 \%)$ and glycopeptides in $n=20$ (43.5).

Almost $n=31(67.4 \%)$ of the patients had had a prior culture growing a multidrug-resistant organism in the last six months. The most commonly found organism was carbapenem-resistant Klebsiella pneumonia in $\mathrm{n}=13(28.2 \%)$, followed by multidrug-resistant Acinetobacter and Pseudomonas aeruginosa $\mathrm{n}=11$ $(23.9 \%)$ of patients. Approximately $n=35(76.1 \%)$, had a urinary catheter in place, and central lines were present in $n=22(47.8 \%)$, surgical drains in $n=14(30.4 \%)$, and VP shunt in $n=1(2.2 \%)$ of patients. There were 29 patients $(63 \%)$ with this infection who had a recent history (past six months) of invasive procedures. Of those, $n=9(31 \%)$ had undergone skin soft tissue procedures, genitourinary surgeries in $\mathrm{n}=8(27.6)$ cases, $\mathrm{n}=7(24.1 \%)$ had abdominal procedures, and $\mathrm{n}=7(24.1 \%)$ had central nervous system interventions.

The most common site of infection was the respiratory tract in $n=13(28.3 \%)$, followed by Urinary tract infections $n=12(26 \%)$, and bloodstream infections $n=12(26.1 \%$.) (Table 1). Approximately $n=21(45.7 \%)$ had respiratory failure, that required mechanical ventilation in $n=11(52.4 \%)$, and non-invasive ventilation in $\mathrm{n}=10(47.6 \%) . \mathrm{N}=17$ patients, $(37 \%)$ were in septic shock shock due to R.terrigena. Of them $\mathrm{n}=13$, $(76.5 \%)$ required vasopressors and $n=4(23.5 \%)$ treated with fluid resuscitation

Monomicrobial growth of Raoultella terrigena was identified from $n=26(56.5 \%)$ of culture specimens, and polymicrobial growth was identified from $n=20(43.5 \%)$ culture specimens. The most common sources were blood in $32.6 \%$, sputum in $28.3 \%$, and urine in $21.7 \%$. The organism was highly resistant to most of the commonly used antibiotics. Carbapenem resistance was present in $91.3 \%$, colistemethate 
resistance $65.2 \%$. Tigecycline resistance present in $10.9 \%$ (checked in 31 strains) and fosfomycin resistance in $28.3 \%$ (checked in 29 strains) (Table-2). In most cases, sensitivities were checked for tigecycline and fosfomycin after they were resistant to colistemethate.

Out of 46 cases, eight patients lost to follow up, and treatment information was not available. The remaining 38 patients, $n=31$ patients $(81.6 \%)$ received combination therapy, and $n=6(15.8 \%)$ received monotherapy. One patient died before starting treatment. The most frequent combination was carbapenem and colistemethate in $n=11(28.9 \%)$, followed by a combination of carbapenem with colistemethate and tigecycline in $n=8(21.1 \%)$ (Table-3). In $71.1 \%$ cases, the treatment was inappropriate for $R$-terrigena while the remaining $28.9 \%$, given appropriately. Mortality association with monotherapy was $(p=0.672)$, and with combination therapy $(p=0.70)$. Out of $n=14(36.84 \%)$ cases in whom repeat cultures for clearance needed, bacteriological clearance was achieved in $n=9(64.2 \%)$ cases. There were 23 cases that needed source control of underlying infection. It was achieved in $n=12(52.1 \%)$. Two cases (5.3\%) relapsed.

The average hospital stay was a median of 11.50 days (IQR=17), with a median of 3 days before positive culture. Approximately $n=23(60.52 \%)$ of patients were seriously ill, requiring intensive care unit care with a median ICU stay of 6 days (IQR=7). In-hospital mortality recorded in $n=17(44.7 \%)$ patients.

In the subgroup analysis of factors associated with death in R.terrigena infections, it was found that chronic kidney disease (CKD) ( $(p=.029)$ and septic shock $(p=.005)$ were significantly associated with mortality. Also, persons with a high (greater or equal to three) Charlson- comorbidity index had increased mortality $(p=0.002)$.

\section{Discussion}

In our study, most patients had hospital-acquired respiratory tract infections, followed by urinary tract infections. Clinical symptoms identified in the vast majority were respiratory failure, septic shock, and altered mental status. The grown isolates were highly resistant to beta-lactams, carbapenems, and colistemethate.

Its clinical impact remained mostly unknown until 2007 when a middle-aged liver transplant person suffered infective endocarditis with R-terrigena (Table-4). Before his surgery for liver transplantation, he was found to be a colonizer with ESBL $R$-terrigena and was given piperacillin-tazobactam as a prophylactic agent. The organism identified after the death of the patient in tissue (aortic valve) ${ }^{4}$. Previously stated that infections caused by the genus Raoultella are common in older-aged groups who were suffering from malignancy or underwent surgical interventions. Many of them developed infections of the biliary tract and had different mortality rates. ${ }^{15}$ In our study group, most patients were male in the middle and older aged group, who were diabetics in the vast majority, followed by CKD and cancer patients. Around two-thirds of them had recent surgical interventions. We had only one patient with biliary 
tract involvement, and we found a higher rate of nosocomial pneumonia and urinary tract infections in our patients. (table-1).

Another case published in 2011 was about a monomicrobial R.terrigena infection. It was causing postlaparotomy intrabdominal sepsis with fluid encasement ${ }^{5}$ (Table-4). The organism identified in blood and drain cultures was sensitive to amoxicillin-clavulanic acid, cephalosporins, ciprofloxacin, and imipenem. The patient was treated successfully with imipenem piperacillin-tazobactam. In 2016, a case of subungual abscess caused by R.terrigena reported, resulting from working in a field. It was pan sensitive and treated with imipenim ${ }^{16}$ (Table-4). However, in most of our patient population, the grown isolates were highly resistant to many drugs, including carbapenems and colistemethate. These findings also present in a recent small case series of three patients published from Pakistan in 2019 (Table-4). In that all three patients were female, two of them had diabetes and one had CKD. All patients had a complicated hospital course; they were already on treatment for other hospital-acquired infections. Two patients had complicated urinary tract infections; they grew highly drug-resistant $R$.terrigena in urine and blood cultures. One died and the other was critically ill and transferred to another hospital. The remaining one patient suffered nosocomial respiratory tract infection by R.terrigena sensitive to colistemethate and tigecycline in both respiratory and blood samples. This patient also died despite appropriate treatment ${ }^{17}$. In comparison, we found a higher frequency of nosocomial pneumonia in our patients. Similarly, we also found increased mortality among our infected cases.

Although our study has a limited sample size and is a single-center experience, it is the largest cohort of patients reported of this rare gram-negative infection to date. In this era of hard to treat hospital-acquired infections and associated antibiotic resistance, we face challenges treating these kinds of pathogens. These emerging opportunistic pathogens and associated drug resistance leave us with a limited choice of antibiotics. Interestingly, we found no outcome difference in treating patients with monotherapy against combination therapy. Patients with many comorbid conditions and immunosuppression can lead to infections with opportunistic organisms, including R.terrigena, leading to increased mortality if not identified and treated correctly.

\section{Limitations}

Because of the scarcity of available resources, we could not detect $R$.terrigena by molecular analysis (standard method).

\section{Conclusion}

Infections caused by R.terrigena are highly drug-resistant, mostly causing hospital-acquired respiratory tract infections that are difficult to treat, leading to prolonged hospital and ICU stays with a high mortality rate. Patients with underlying renal dysfunction, those on vasopressor support, and a high $\mathrm{CCl}$ score are at greater risk of death due to this infection. 


\section{Declarations}

\section{Authors' Contributions:}

1. Ishfaque Ahmed

Data collection, Data analysis, and interpretation, Manuscript writing

2. Nosheen Nasir

Study design and methodology, Questionnaire development, editing of manuscript draft

3. Bushra Jamil

Draft editing and charts/table review

4. Syed Faisal Mahmood- Corresponding author

Study Idea, Abstract writing, and manuscript editing

\section{Competing Interest}

I declare that the authors have no competing interests (Financial or non- financial) as defined by BMC, or other interests that might be perceived to influence the results and/or discussion reported in this paper.

\section{Ethical approval and consent to participate:}

Our study was reviewed and approved as exemption by Aga Khan University (AKU) ethics review committee (Refrence number: 2019-1232-3287). The study was performed in accordance with the Declaration of Helsinki and guidelines and regulations of Aga Khan University ethics review committee. The requirement for written informed consent was waived as the data was collected retrospectively from medical records and was de-identified, which had been approved by the the Aga Khan University (AKU) ethics reveiw committee.

\section{Consent for publication:}

Not applicable

\section{Animal Ethics:}


We confirm that all experimental protocols involving animals were approved by the Aga Khan University licensing committee and all methods were carried out in accordance with ARRIVE guidelines.

\section{Availability of data and material:}

The datasets generated and/or analyzed during the current study are not publicly available due to institutional policy but are available from the corresponding author on reasonable request.

\section{Funding:}

Not applicable

\section{Acknowledgment:}

Not applicable

\section{References}

1. Izard D, Ferragut C, Gavini F, Kersters K, De Ley J, Leclerc HJIJoS, et al. Klebsiella terrigena, a new species from soil and water. Int J Syst Bacteriol 1981; https://doi.org/10.1099/00207713-31-2-116

2. Drancourt M, Bollet C, Carta A, Rousselier PJljos, microbiology e. Phylogenetic analyses of Klebsiella species delineate Klebsiella and Raoultella gen. nov., with description of Raoultella ornithinolytica comb. nov., Raoultella terrigena comb. nov. and Raoultella planticola comb. nov. Int J Syst Evol Microbiol. 2001; https://doi.org/10.1099/00207713-51-3-925

3. Ponce-Alonso M, Rodriguez-Rojas L, Del Campo R, Canton R, Morosini MI. Comparison of different methods for identification of species of the genus Raoultella: report of 11 cases of Raoultella causing bacteraemia and literature review. Clin Microbiol Infect. 2016; https://doi.org/10.1016/j.cmi.2015.10.035

4. Goegele H, Ruttmann E, Aranda-Michel J, Kafka R, Stelzmueller I, Hausdorfer H, et al. Fatal endocarditis due to extended spectrum betalactamase producing Klebsiella terrigena in a liver transplant recipient. Wien Klin Wochenschr. 2007; https://doi.org/10.1007/s00508-007-0812-5

5. Shaikh MM, Morgan M. Sepsis caused by Raoultella terrigena. JRSM short reports. 2011; https://doi.org/10.1258/shorts.2011.010127

6. Chun S, Yun JW, Huh HJ, Lee NY. Clinical characteristics of Raoultella ornithinolytica bacteremia. Infection. 2015; https://doi.org/10.1007/s15010-014-0696-z

7. Tseng SP, Wang JT, Liang CY, Lee PS, Chen YC, Lu PL. First Report of bla(IMP-8) in Raoultella planticola. Antimicrobial agents and chemotherapy. 2014; https://doi.org/10.1128/aac.00231-13 
8. Chun S, Yun JW, Huh HJ, Lee NY. Low virulence? Clinical characteristics of Raoultella planticola bacteremia. Infection. 2014; https://doi.org/10.1007/s15010-014-0664-7

9. Kim SH, Roh KH, Yoon YK, Kang DO, Lee DW, Kim MJ, et al. Necrotizing fasciitis involving the chest and abdominal wall caused by Raoultella planticola. BMC Infect Dis. 2012; https://doi.org/10.1186/1471-2334-12-59

10. K OC, Kelly J, Niriain U. A Rare Case of Soft-Tissue Infection Caused by Raoultella planticola. Case Rep Med. 2010; https://doi.org/10.1155/2010/134086

11. Olson DS, Jr., Asare K, Lyons M, Hofinger DM. A novel case of Raoultella planticola urinary tract infection. Infection. 2013; https:// doi.org/10.1007/s15010-012-0294-x

12. Yokota K, Gomi H, Miura Y, Sugano K, Morisawa Y. Cholangitis with septic shock caused by Raoultella planticola. J Med Microbiol. 2012; https://doi.org/10.1099/jmm.0.032946-0

13. Teo I, Wild J, Ray S, Chadwick DJCrim. A rare case of cholecystitis caused by Raoultella planticola. Case Rep Med. 2012; https://doi.org/10.1155/2012/601641

14. Alves MS, Riley LW, Moreira BM. A case of severe pancreatitis complicated by Raoultella planticola infection. J Med Microbiol. 2007; https://doi.org/10.1099/jmm.0.46889-0

15. Ershadi A, Weiss E, Verduzco E, Chia D, Sadigh M. Emerging pathogen: a case and review of Raoultella planticola. Infection. 2014; https://doi.org/10.1007/s15010-014-0638-9

16. Wang Y, Jiang X, Xu Z, Ying C, Yu W, Xiao Y. Identification of Raoultella terrigena as a Rare Causative Agent of Subungual Abscess Based on 16S rRNA and Housekeeping Gene Sequencing. Can J Infect Dis Med Microbiol. 2016; https://doi.org/10.1155/2016/3879635

17. Mal PB, Sarfaraz S, Herekar F, Ambreen R. Clinical manifestation and outcomes of multi-drug resistant (MDR) Raoultella terrigena infection - A case series at Indus Health Network, Karachi, Pakistan. IDCases. 2019; https://doi.org/10.1016/j.idcr.2019.e00628

\section{Tables}




\section{Table-1}

\section{Clinical features and outcomes of R.terrigena}

Age in years:

Sex:

Site of infection:

\begin{tabular}{ll} 
Pneumonia & $\mathrm{n}=9$ \\
Tracheitis & $\mathrm{n}=4$ \\
Cystitis & $\mathrm{n}=6$ \\
Pyelonephritis & $\mathrm{n}=6$ \\
CLABSI & $\mathrm{n}=5$ \\
\hline Unspecified BSI & $\mathrm{n}=2$ \\
Necrotizing fasciitis & $\mathrm{n}=2$ \\
\hline Bed sore infection & $\mathrm{n}=1$ \\
Cellulitis & $\mathrm{n}=1$ \\
\hline Septic arthritis & $\mathrm{n}=1$ \\
Ventriculitis & $\mathrm{n}=1$ \\
\hline Peritonitis & $\mathrm{n}=1$
\end{tabular}

Clinical presentation:

Hypotension (Systolic $<90$ )

Respiratory failure

Altered mental status:

Central lines:

VP shunt:

Surgical drains:

Folly's

Prior antibiotics (6, months)
Mean: (57.72), (S.E: 2.695)

$M:(n=28), F:(n=18)$

M: (60.9\%), F (39.1\%)
Median: (61.50) IQR = 28

$(19.6 \%)$

$(8.7 \%)$

$(13 \%)$

$(10.9 \%)$

(4.3\%)

(4.3\%)

(2.2\%)

(2.2\%)

(2.2\%)

(2.2\%)

(2.2\%)

(23.6\%)

(29.2\%)

(54.34\%)

(47.8\%)

(2.2\%)

(30.4\%)

(76.1\%)

(78.3\%) 


\section{Table-1}

\section{Clinical features and outcomes of R.terrigena}

\begin{tabular}{|c|c|c|}
\hline Prior hospitalization: (6, months) & $\mathrm{n}=34$ & $(73.9 \%)$ \\
\hline Prior MDRO's (6, months) & $\mathrm{n}=31$ & $(67.4 \%)$ \\
\hline Recent procedures: & $\mathrm{n}=29$ & $(63 \%)$ \\
\hline Skin, soft tissues & $n=9$ & $(31 \%)$ \\
\hline Abdomen & $n=7$ & $(24.1 \%)$ \\
\hline CNS & $n=7$ & $(24.1 \%)$ \\
\hline Genitourinary & $\mathrm{n}=8$ & $(27.6 \%)$ \\
\hline Stent placement & $\mathrm{n}=4$ & $(13.8 \%)$ \\
\hline Chest: & $\mathrm{n}=4$ & $(13.8 \%)$ \\
\hline Others: & $\mathrm{n}=6$ & $(20.7 \%)$ \\
\hline \multicolumn{3}{|l|}{ Co-morbids: } \\
\hline Diabetes mellitus & $\mathrm{n}=18$ & $(39.1 \%)$ \\
\hline Chronic kidney disease & $\mathrm{n}=16$ & $(34.7 \%)$ \\
\hline Malignancy & $\mathrm{n}=11$ & $(23.9 \%)$ \\
\hline Cerebrovascular accident & $\mathrm{n}=7$ & $(15.2 \%)$ \\
\hline Chronic liver diseae & $\mathrm{n}=8$ & $(17.3)$ \\
\hline Steroid therapy & $\mathrm{n}=2$ & $(4.3 \%)$ \\
\hline Connective tissue disease & $\mathrm{n}=1$ & $(2.1 \%)$ \\
\hline Hospital stay (days) & Mean (18.47) & Median (11.50) IQR = 17 \\
\hline ICU stay (days) & Mean $(7.70)$ & Median (6) IQR = 7 \\
\hline Alive & $\mathrm{n}=21$ & $(55.3 \%)$ \\
\hline Dead & $\mathrm{n}=17$ & $(44.7 \%)$ \\
\hline
\end{tabular}




\section{Table-2}

\section{Drugs susceptibility of R-terrigena}

\begin{tabular}{|lllll|}
\hline Antibiotics & Sensitivity & Resistance & Intermediate & Unchecked \\
\hline Amoxi-clav & $2.2 \%$ & $93.5 \%$ & $2.2 \%$ & $2.2 \%$ \\
\hline Amikacin & $19.6 \%$ & $76.1 \%$ & $2.2 \%$ & $2.2 \%$ \\
\hline Imipenim & $4.3 \%$ & $89.1 \%$ & $2.2 \%$ & $4.3 \%$ \\
\hline Piper-tazo & $4.3 \%$ & $91.3 \%$ & $2.2 \%$ & $2.2 \%$ \\
\hline Gentamicin & $8.7 \%$ & $89.1 \%$ & $2.2 \%$ & \\
\hline Ceftriaxone & $2.2 \%$ & $97.8 \%$ & & \\
\hline Co-trimoxazole & $8.7 \%$ & $89.1 \%$ & & $2.2 \%$ \\
\hline Ciprofloxacin & $6.5 \%$ & $91.3 \%$ & & $2.2 \%$ \\
\hline Meropenem & $8.7 \%$ & $91.3 \%$ & & $10.9 \%$ \\
\hline Colistemethate & $23.9 \%$ & $65.2 \%$ & & $32.6 \%$ \\
\hline Tigecyclin & $30.4 \%$ & $10.9 \%$ & $26.1 \%$ & $37 \%$ \\
\hline Fosfomycin & $15.2 \%$ & $28.3 \%$ & $19.6 \%$ & \\
\hline
\end{tabular}




\section{Table-3}

Frequently used antibiotics as combination therapy

\begin{tabular}{|lll|}
\hline Meropenem, colistemethate & $\mathrm{n}=11$ & $(28.9 \%)$ \\
\hline Meropenem, colistemethate, tigecycline & $\mathrm{n}=8$ & $(21.1 \%)$ \\
\hline Meropenem, tigecycline & $\mathrm{n}=2$ & $(5.3 \%)$ \\
Meropenem, colistemethate, ciprofloxacin & $\mathrm{n}=1$ & $(2.6 \%)$ \\
\hline Tigecycline, colistemethate & $\mathrm{n}=1$ & $(2.6 \%)$ \\
\hline Tigecycline, fosfomycin & $\mathrm{n}=1$ & $(2.6 \%)$ \\
\hline Imipenim, colistemethate & $\mathrm{n}=1$ & $(2.6 \%)$ \\
\hline Meropenem, colistemethate, fosfomycin & $\mathrm{n}=1$ & $(2.6 \%)$ \\
\hline Fosfomycin, moxifloxacin & $\mathrm{n}=1$ & $(2.6 \%)$ \\
\hline Imipenim, colistemethate, fosfomycin & $\mathrm{n}=1$ & $(2.6 \%)$ \\
\hline Amikacin, levofloxacin & $\mathrm{n}=1$ & $(2.6 \%)$ \\
\hline Ceftazidime, piptaz & $\mathrm{n}=1$ & $(2.6 \%)$ \\
\hline
\end{tabular}




\section{Table-4}

Previously published case reports and case series

\begin{tabular}{lllll} 
Authors & $\begin{array}{l}\text { Case } \\
\text { numbers }\end{array}$ & $\begin{array}{l}\text { Infection } \\
\text { Source }\end{array}$ & Treatment & Outcome \\
$\begin{array}{l}\text { Goegele H. } \\
\text { et. } \mathrm{al}^{4}\end{array}$ & 1 & $\begin{array}{l}\text { Tissue (aortic } \\
\text { valve) }\end{array}$ & No & Death \\
\hline
\end{tabular}

$\begin{array}{llll}\begin{array}{l}\text { Oslon Ds Jr. } \\ \text { et. al. }{ }^{11}\end{array} & 1 & \text { Body fluid } & \begin{array}{l}\text { Piperacillin Tazobactam, } \\ \text { Imipenem }\end{array}\end{array}$

$\begin{array}{lllll}\text { Wang Y. et. } & 1 & \text { Pus } & \text { Imipenem }\end{array}$
al. $^{20}$

Mal PB. Et. 3 (Case Urine

al. $^{15}$

1)

Fosfomycin, Tigecycline, Co-

Death trimoxazole

$\begin{array}{llll}\text { (Case 2) } & \begin{array}{l}\text { Tracheal } \\ \text { aspirate }\end{array} & \begin{array}{l}\text { Colistemethate, Tigecycline, } \\ \text { Co-trimoxazole }\end{array} & \text { Death } \\ \text { (Case 3) } & \text { Urine } & \text { Fosfomycin } & \begin{array}{l}\text { Transferred to } \\ \text { another hospital. }\end{array}\end{array}$

\section{Figures}

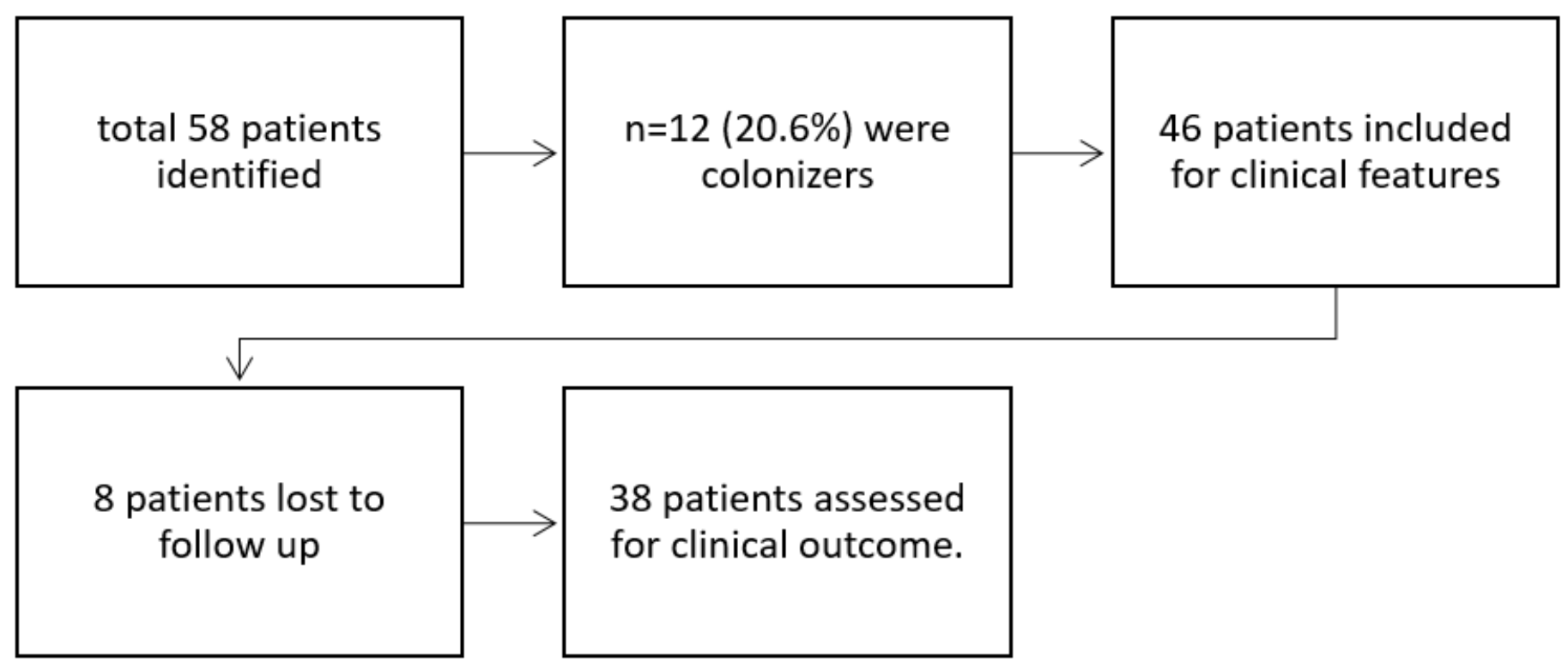




\section{Figure 1}

Flow chart showing the number of patients in the study 